\title{
Integration of a non-contact temperature sensor in the terminal using remote monitoring of readings
}

\author{
Arseniy Vasilenko*, Svetlana Studennikova, and Albina Agibalova \\ Don State Technical University, Rostov-on-Don, 344000, Russia
}

\begin{abstract}
The article is devoted to an overview of the project aimed at optimizing the production process in a wide range of enterprises. Considered is the creation of an installation that implements the automation of measuring the temperature of people at checkpoints with subsequent remote monitoring of readings. The result of the development involves an increase in the level of safety of employees and the elimination of the "human factor". The value of the carbon footprint generated by the device during the year was, of $\mathrm{CO} 2$, respectively. At the same time, the transition to the use of energy-saving modes in IoT devices potentially reduces energy consumption and the value of the carbon footprint by more than 1000 times.
\end{abstract}

\section{Introduction}

Today, many institutions are equipped with checkpoints in the form of turnstiles. During the pandemic associated with the spread of the COVID-19 virus, it became necessary to monitor the temperature of those entering the premises. For this, performers are appointed in the person of employees of this institution or third parties. Measurements are made using non-contact thermometers. The specified project contains a temperature sensor, which also does not require contact with the surface. However, it has a higher speed than devices on the market.

Automation of monitoring the state of people (in particular, body temperature) passing through checkpoints is one of the modern trends in improving security. Modern measuring instruments can potentially replace a person with an electronic thermometer, and can be installed in access turnstiles, as well as integrated into the enterprise information system. If the temperature of a person deviates from the medical standard, the turnstile will be automatically blocked.

This project is designed, first of all, to reduce morbidity due to the exclusion of the "human factor". For the same reason, taking into account the measurement speed, increased throughput at checkpoints and a decrease in enterprise costs will be provided. In the future, it is possible to implement employee identification in order to monitor his condition.

* Corresponding author: arseny.vasilenko@aiesec.net 
At the same time, the project is designed to reduce emissions of carbon dioxide into the atmosphere due to its relatively low capacity. The use of such technologies makes it possible to reduce the level of such emissions by more than 1000 times.

\section{Methods and materials}

\subsection{Physical organization of the project}

The object of research is based on NodeMCU Wemos V3 with ESP8266 microcontroller [1]. This microprocessor supports RTOS and operates at $80 \mathrm{MHz}$ to $160 \mathrm{MHz}$ adjustable clock frequency. NodeMCU has $128 \mathrm{~KB}$ RAM and $4 \mathrm{MB}$ of Flash memory to store data and programs. Its high processing power with in-built Wi-Fi / Bluetooth and Deep Sleep Operating features make it ideal for IoT projects.

NodeMCU can be powered using Micro USB jack and VIN pin (External Supply Pin). It supports UART, SPI, and I2C interface.

Temperature measurements is taken by MLX90614 [2]. The MLX90614 is an infrared thermometer for non-contact temperature measurements. Both the IR sensitive thermopile detector chip and the signal conditioning ASIC are integrated in the same TO-39 can. Integrated into the MLX90614 are a low noise amplifier, 17-bit ADC and powerful DSP unit thus achieving high accuracy and resolution of the thermometer. The thermometer comes factory calibrated with a digital SMBus output giving full access to the measured temperature in the complete temperature range(s) with a resolution of $0.02^{\circ} \mathrm{C}$. The user can configure the digital output to be pulse width modulation (PWM). As a standard, the 10-bit PWM is configured to continuously transmit the measured temperature in range of -20 to $120^{\circ} \mathrm{C}$, with an output resolution of $0.14^{\circ} \mathrm{C}$.

An infrared sensor is used to detect obstacles [3]. The TCRT5000 can detect color and distance. The sensor emits IR rays and then detects if it receives a reflection. This sensor is often used in line following robots, edge detection. The sensor allows you to sense white or black surfaces by reading the analog value from it. The measuring distance is between 1 and $8 \mathrm{~mm}$. The onboard potentiometer can be used to adjust the sensitivity.

TM1637 indicator is used for the temperature indication [4]. On the front of the TM1637 module, there is a four-digit 7-segment display with a colon in the center, on the back there is a TM1637 chip from Titan Micro Electronics. The 7-segment display itself consists of seven LEDs, designated by letters: from "a" to "g", which light up independently of each other and can display numbers and letters. Also, the board is 3.3 - 5V compatible, no additional resistors or capacitors are required.

For additional graphical visualization there is a light strip WS2812. WS2812B is a intelligent control LED light source that the control circuit and RGB chip are integrated in a package of 5050 components. It internal include intelligent digital port data latch and signal reshaping amplification drive circuit. Also include a precision internal oscillator and a $12 \mathrm{~V}$ voltage programmable constant current control part, effectively ensuring the pixel point light color height consistent. The data transfer protocol use single NRZ communication mode. After the pixel power-on reset, the DIN port receive data from controller, the first pixel collect initial 24bit data then sent to the internal data latch, the other data which reshaping by the internal signal reshaping amplification circuit sent to the next cascade pixel through the DO port. After transmission for each pixel, the signal to reduce 24bit. pixel adopt auto reshaping transmit technology, making the pixel cascade number is not limited the signal transmission, only depend on the speed of signal transmission. LED with low driving voltage, environmental protection and energy saving, high brightness, 
scattering angle is large, good consistency, low power, long life and other advantages. The control chip integrated in LED above becoming more simple circuit, small volume, convenient installation.

For voltage conversion from power supply the dc-dc step-down regulator is used. Mini360 (MH-Mini-360 / DSN-Mini-360 / HX-Mini-360) is a DC-DC switching voltage regulator with high efficiency (up to 96\%). The module is built on the MP2307 chip (MP2307DN). The Mini-360 converter is capable of operating with an applied voltage from 4.75 to $23 \mathrm{~V}$. Due to the high switching frequency $(340 \mathrm{KHz})$, it allows to obtain a large current in the load (up to $1.8 \mathrm{~A}$ continuously, up to $3 \mathrm{~A}$ for a short time) with a small converter size. The no-load output ripple is less than $30 \mathrm{mV}$. When setting currents close to the maximum value, and long-term operation with such values, an additional heatsink should be installed.

For controlling the turnstile the relay module is used. It is a 1-channel relay interface board, which can be controlled directly by a wide range of microcontrollers. It is also able to control various appliances and other equipment with large current. Relay output maximum contact is AC250V $10 \mathrm{~A}$ and DC30V 10A. Standard interface can be directly connected with microcontrollers. The working status indicator light is conducive to the safe use.

Widely used for all MCU control, industrial sector, PLC control, smart home control.

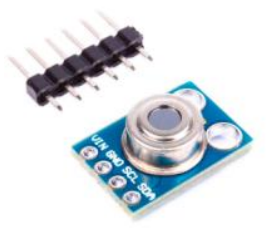

MLX90614

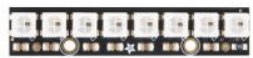

WS2812

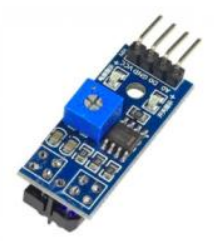

IR-sensor

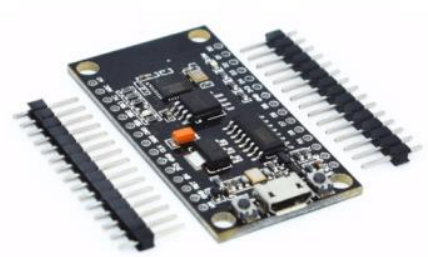

NodeMCU Wemos V3

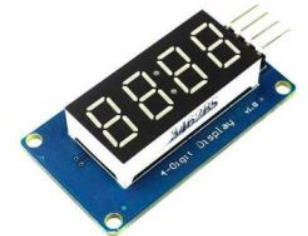

TM1637

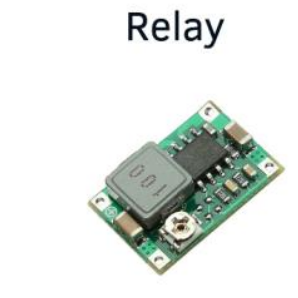

DC-DC regulator

Fig. 1. Component devices

The wiring diagram is shown in Fig. 2. 


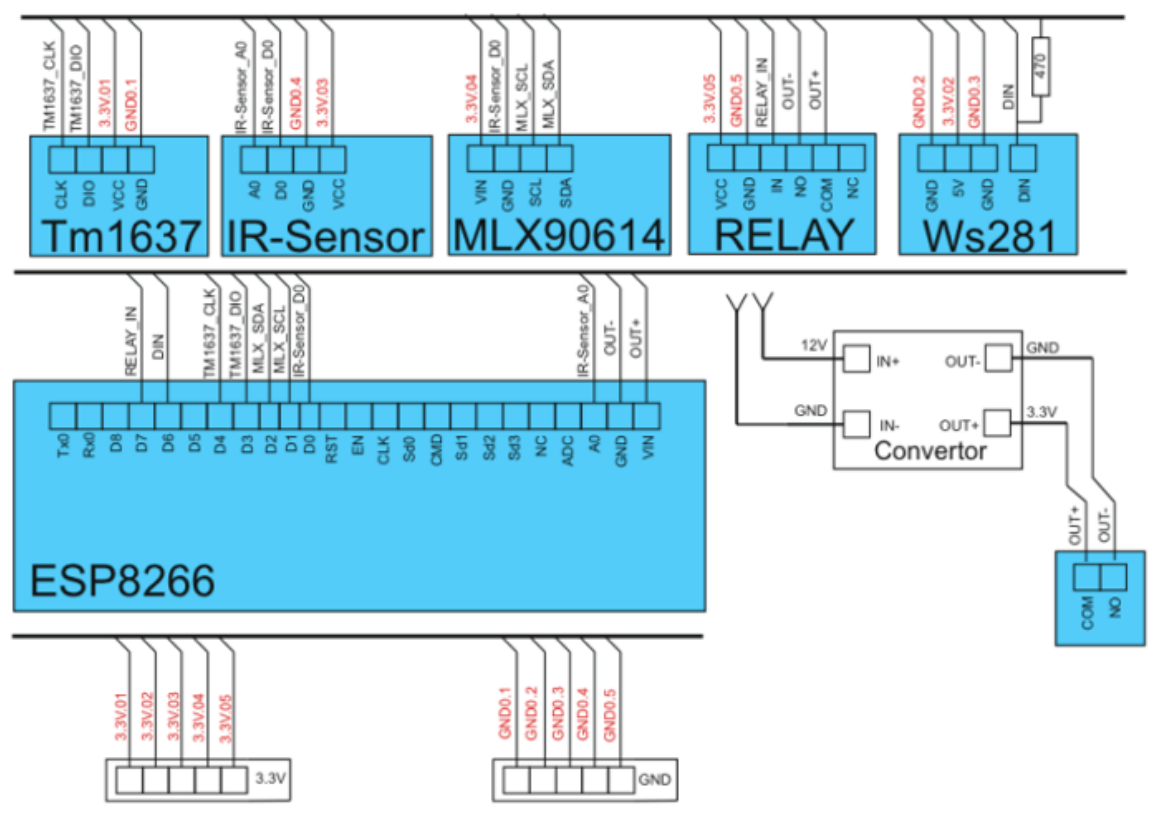

Fig. 2. Wiring diagram

The structural diagram of the project is as follows in Fig. 3.

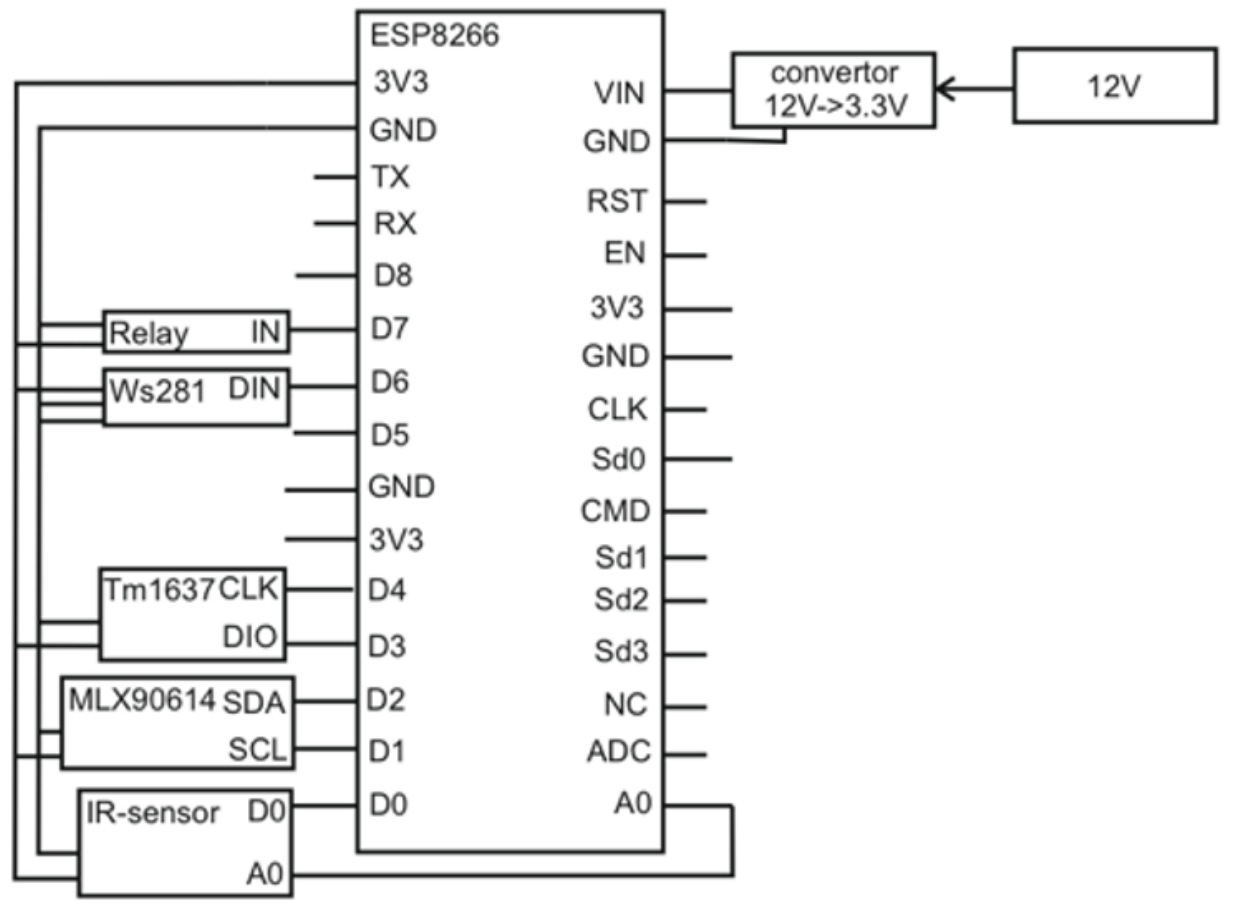

Fig. 3. Block diagram of connections 
The current appearance of the project is shown in Fig. 4.

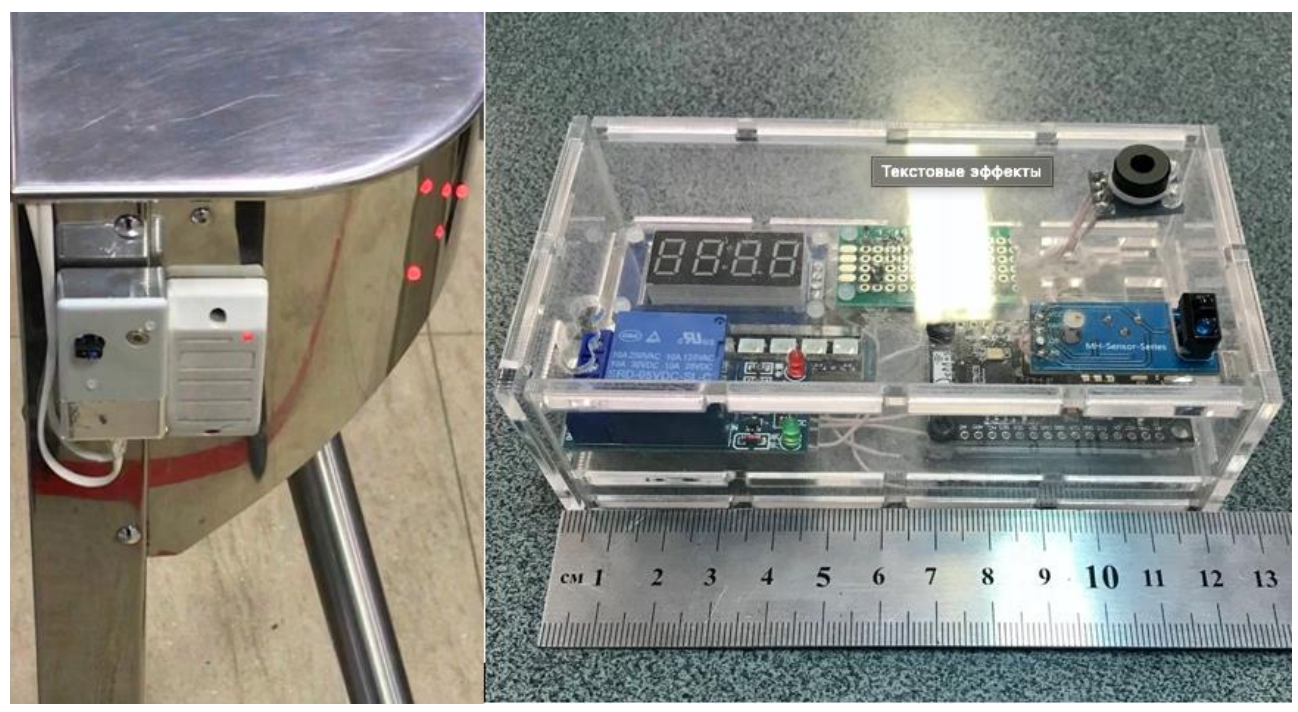

Fig. 4. Layout and appearance of the project

\subsection{Network communication technologies used in the study}

\subsubsection{MQTT}

MQTT is a pub / sub messaging protocol. A communication system built on MQTT consists of a publisher server, a broker server, and one or more clients. The publisher does not require any adjustments to the number or location of subscribers who receive messages. In addition, subscribers do not need to be configured for a specific publisher. There may be multiple brokers in the system that distribute messages.

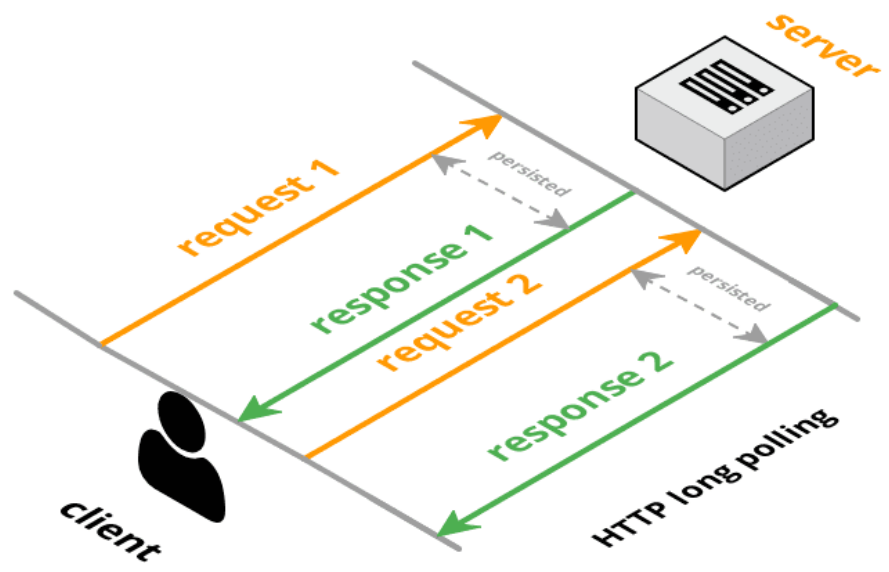

Fig. 5. Graphical representation of MQTT-protocol 
MQTT provides a way to create a hierarchy of communication channels - a kind of branch with leaves. Whenever a publisher has new data to distribute to customers, the message is accompanied by a shipping control note [5]. Higher-level clients can receive every message, while lower-level clients can receive messages related to only one or two basic channels that branch off at the bottom of the hierarchy. This facilitates the exchange of information ranging in size from two bytes to 256 megabytes.

\subsection{Logical organization of project}

To implement the software part of the project, the Arduino IDE development environment was chosen. The algorithm of work is as follows: a person approaches the terminal, raises his palm at a distance of up to 10 centimeters from the infrared proximity sensor IR-sensor, as a result of the instantaneous operation of which, the signal is transmitted to the ESP8266 microcontroller (aka NodeMCU Wemos V3) and the MLX90614 infrared temperature sensor at the same moment reads the degree from the palm surface. Depending on the temperature entering or leaving the medical norm, MK sends a signal to a relay connected to the turnstile. If the temperature is acceptable, the entrance can be made by a pass, otherwise the turnstile will be blocked. Each temperature measurement is time-bound and stored on the MQTT server [6]. The following connection is demonstrated with application in Fig.6.

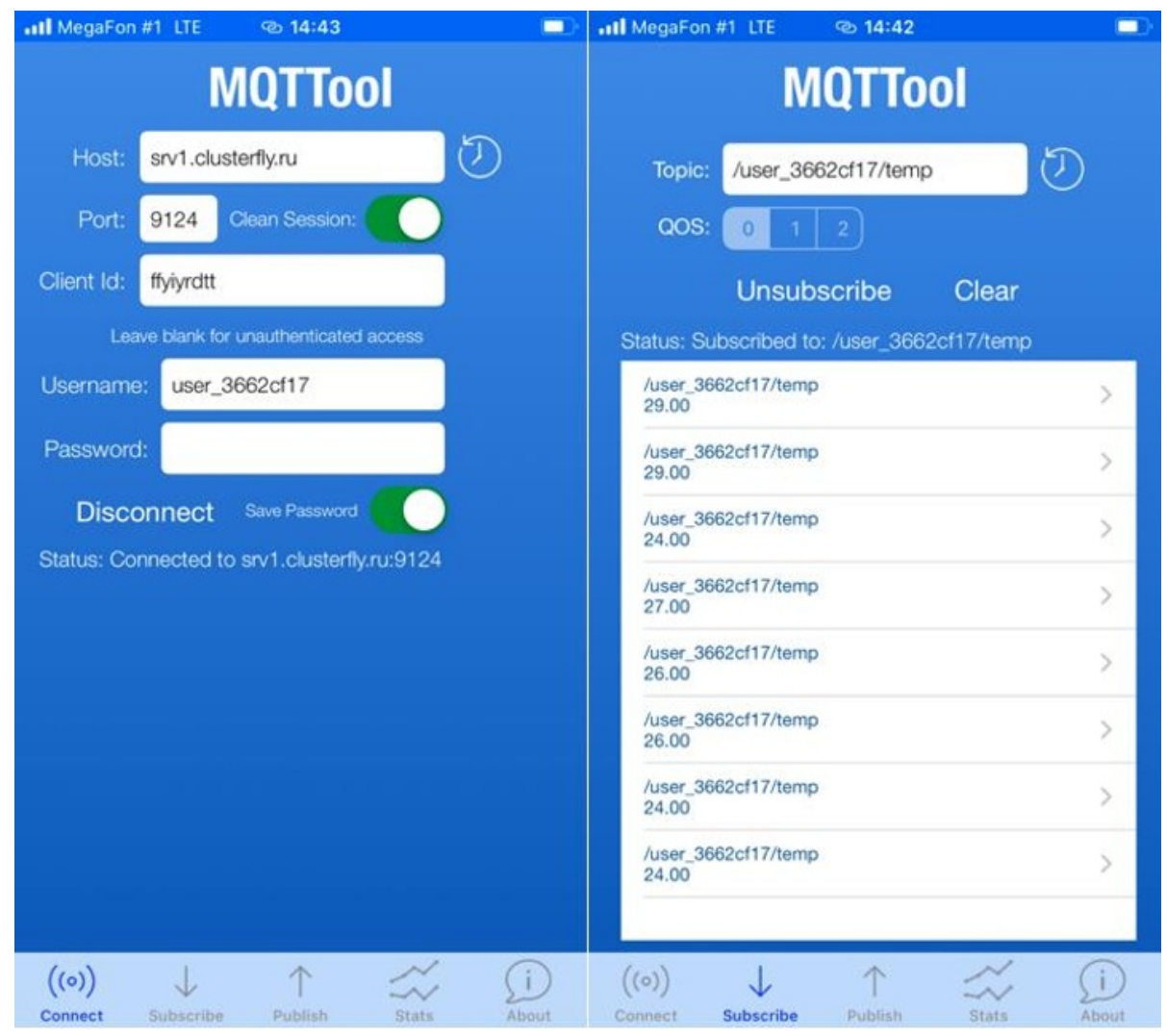

Fig. 6. Monitoring app and demo 


\section{Results and discussion}

The experiments were conducted on the Laboratory of Local Automation and Embedded Systems of the Department "Automation of Production Processes", Don State Technical University. Then the project was tested in real conditions at one of the university's checkpoints. The results include an increase in throughput due to the speed of measurements, a decrease in the risk of infection due to the absence of a hired worker with a thermometer at the point, which in addition excludes the influence of the "human factor". Moreover, a person, on average per year, emits up to 400 kilograms of carbon dioxide [7], which is significantly higher than that of this device. To make sure of this, we will carry out the calculation as follows: the power allocated by the project is $0.1 \mathrm{~W}$. Let's assume that it works around the clock throughout the year. The specific gravity of carbon dioxide, equivalent to $1 \mathrm{~kW} * \mathrm{~h}$, is 0.325 kilograms [8]. In this case, a total of 284.7 grams will be allocated for the year, which is more than 1000 times less than that of a person.

\section{Conclusion}

Despite the analogs of temperature reading available abroad from a person entering the room, they have a significant advantage of a face scanner that stores data in the database. However, this project is more universal, since reading a person with subsequent saving is not allowed everywhere. Moreover, the cost of this project will ultimately turn out to be significantly lower than the existing competitors, which will increase its availability for a wide range of consumers. The device is mobile and, with proper design, can be ergonomically integrated into any terminal.

The value of the carbon footprint generated by the device during the year was, 284,7 $\mathrm{g}$. At the same time, the transition to the use of energy-saving modes in IoT devices potentially reduces energy consumption and the value of the carbon footprint by more than 1000 times [9].

This research was supported by Boldyrev Aleksander and Lukyanov Aleksander, who provided information and experiences that greatly aided the study, although they may partially disagree with conclusions of this document.

\section{References}

1. A. Parsa, T. A. Najafabadi and F. R. Salmasi, Implementation of smart optimal and automatic control of electrical home appliances (IoT), (2017)

2. P. Asopa, P. Purohit, R. R. Nadikattu and P. Whig, ICICV (2021)

3. Green Energy and Networking: 7th EAI International Conference, GreeNets 2020, Harbin, China, (2020)

4. Portales, Raul. Android Things Quick Start Guide: Build Your Own Smart Devices Using the Android Things Platform. UK, (2018)

5. Monika Kashyap, Vidushi Sharma, Neeti Gupta, Taking MQTT and NodeMcu to IoT: Communication in Internet of Things, (2018)

6. J. Mesquita, D. Guimarães, C. Pereira, F. Santos and L. Almeida, (ETFA), (2018)

7. U.S. Energy Information Administration. URL: https://www.eia.gov/tools/faqs/faq.php?id=74

8. P. Asopa, P. Purohit, R. R. Nadikattu and P. Whig, (ICICV), (2021) 
9. Shovic, John. Introduction to the ESP8266 and the IoT: Projects with WiFi and the Arduino IDE. USA, (2016) 